\title{
Multi-point boundary value problems for an increasing homeomorphism and positive homomorphism on time scales
}

Liu Yang ${ }^{1,2^{*}}$ and Weiguo Zhang ${ }^{1}$

\footnotetext{
* Correspondence: yliu1219@163. com

${ }^{1}$ College of Science, University of Shanghai for Science and

Technology, Shanghai, 200093, P.R.

China

Full list of author information is available at the end of the article
}

\begin{abstract}
Investigated here are interesting aspects of the positive solutions for two kinds of $m$ point boundary value problems for an increasing homeomorphism and positive homo-morphism on time scales. By using the Avery-Peterson fixed point theorem, we obtain the existence of at least three positive solutions for these problems. The interesting point is that the nonlinear term depends on the first-order deltaderivative explicitly.
\end{abstract}

Keywords: boundary value problem, time scale, fixed point, cone, increasing homeomorphism and positive homomorphism

\section{Introduction}

With the development of boundary value problems for differential equations [1-5], difference equations [6,7], and the theory of time scales [8-12], the existence of solutions for boundary value problems on time scales have attracted many author's attention. Recently in [13], the authors considered positive solutions for boundary value problem of the following second-order dynamic equations on time scales

$$
\begin{aligned}
& \left(\phi\left(u^{\Delta}\right)\right)^{\nabla}+a(t) f(t, u(t))=0, \quad t \in(0, T), \\
& u(0)=\sum_{i=1}^{m-2} \alpha_{i} u\left(\xi_{i}\right) \phi\left(u^{\Delta}(T)\right)=\sum_{i=1}^{m-2} \beta_{i} \phi\left(u^{\Delta}\left(\xi_{i}\right)\right),
\end{aligned}
$$

where $\varphi: R \rightarrow R$ is an increasing homeomorphism and positive homomorphism and $\varphi(0)=0$. Here a projection $\varphi: R \rightarrow R$ is called an increasing homeomorphism and homomorphism, if the following conditions are satisfied:

(i) if $x \leq y$, then $\varphi(x) \leq \varphi(y), \forall x, y \in R$;

(ii) $\varphi$ is a continuous bijection and its inverse mapping is also continuous;

(iii) $\varphi(x y)=\varphi(x) \varphi(y), \forall x, y \in R$.

By using a fixed point theorem, they obtained an existence theorem for positive solutions for this problem. In [14], Han and Jin established existence results of positive solutions for problem $(1.1,1.2)$ by using fixed point index theory. Sang et al. [15]

(c) 2012 Yang and Zhang; licensee Springer. This is an Open Access article distributed under the terms of the Creative Commons Attribution License (http://creativecommons.org/licenses/by/2.0), which permits unrestricted use, distribution, and reproduction in any medium, provided the original work is properly cited. 
considered the problem

$$
\begin{aligned}
& \left(\phi\left(u^{\Delta}\right)\right)^{\nabla}+a(t) f(t, u(t))=0, \quad t \in(0, T) \\
& \phi\left(u^{\Delta}(0)\right)=\sum_{i=1}^{m-2} \alpha_{i} \phi\left(u^{\Delta}\left(\xi_{i}\right)\right), u(T)=\sum_{i=1}^{m-2} \beta_{i} u\left(\xi_{i}\right) .
\end{aligned}
$$

By using a fixed point index theorem, the existence results of positive solutions for this problem were established.

However, the nonlinear terms $f$ in [13-15] does not depend on the first order delta derivative. It is well-known that many difficulties occur when the nonlinear term $f$ depends on the first order delta derivative explicitly. To the author's best knowledge, positive solutions are not available for the case when the boundary value problem for an increasing homeomorphism and positive homomorphism on a time scale in which the nonlinear term depends on the first order delta derivative. This article will fill this gap in the literature. In this article, we consider the existence of positive solutions for the second-order nonlinear $m$-point dynamic equation on a time scale with an increasing homeomorphism and positive homomorphism,

$$
\begin{aligned}
& \left(\phi\left(u^{\Delta}\right)\right)^{\nabla}+a(t) f\left(t, u(t), u^{\Delta}(t)\right)=0, \quad t \in(0, T) \\
& u(0)=\sum_{i=1}^{m-2} \alpha_{i} u\left(\xi_{i}\right), \phi\left(u^{\Delta}(T)\right)=\sum_{i=1}^{m-2} \beta_{i} \phi\left(u^{\Delta}\left(\xi_{i}\right)\right) \text { or } \\
& \phi\left(u^{\Delta}(0)\right)=\sum_{i=1}^{m-2} \alpha_{i} \phi\left(u^{\Delta}\left(\xi_{i}\right)\right), u(T)=\sum_{i=1}^{m-2} \beta_{i} u\left(\xi_{i}\right)
\end{aligned}
$$

where $\xi_{i} \in T_{k}^{k}$ for $i \in\{1,2, \ldots, m-2\}, T$ is a time scale.

We will assume that the following conditions are satisfied throughout this:

(H1) $\alpha_{i}, \beta_{i} \in[0,+\infty)$ satisfy $0<\sum_{i=1}^{m-2} \alpha_{i}<1, \quad 0<\sum_{i=1}^{m-2} \beta_{i}<1$.

(H2) $f \in[0, T] \times[0, \infty) \times R \rightarrow[0, \infty)$ is continuous.

Our main results will depend on an application of a fixed point theorem due to Avery and Peterson which deals with fixed points of a cone-preserving operator defined on an ordered Banach space. By using analysis techniques and the Avery-Peterson fixed point theorem, we obtain sufficient conditions for existence of at least three positive solutions of the problems $(1.5,1.6)$ and $(1.5,1.7)$.

\section{Preliminaries}

First we present some basic definitions on time scales which can be found in Atici and Guseinov [8].

A time scale $T$ is a closed nonempty subset of $R$. For $t<\sup T$ and $r>\inf T$, we define the forward jump operator $\sigma$ and the backward jump operator $\rho$ respectively by

$$
\begin{aligned}
& \sigma(t)=\inf \{\tau \in T \mid \tau>t\} \in T, \\
& \rho(r)=\sup \{\tau \in T \mid \tau<r\} \in T,
\end{aligned}
$$


for all $t \in T$. If $\sigma(t)>t, \mathrm{t}$ is said to be right scattered, and if $\sigma(t)=t, t$ is said to be right dense. If $\rho(t)<t$, $\mathrm{t}$ is said to be left scattered, and if $\rho(t)=t, t$ is said to be left dense. A function $f$ is left-dense continuous, if $f$ is continuous at each left dense point in $\mathrm{T}$ and its right-sided limits exists at each right dense points.

For $u: T \rightarrow R$ and $t \in T$, we define the delta derivative of $u(t), u^{\Delta}(t)$, to be the number (when it exists), with the property that for each $\varepsilon>0$, there is a neighborhood $U$ of $t$ such that

$$
\left|u(\sigma(t))-u(s)-u^{\Delta}(t)(\sigma(t)-s)\right| \leq \varepsilon|\sigma(t)-s|,
$$

for all $s \in U$.

For $u: T \rightarrow R$ and $t \in T$, we define the nabla derivative of $u(t), u^{\nabla}(t)$, to be the number (when it exists), with the property that for each $\varepsilon>0$, there is a neighborhood $U$ of $t$ such that

$$
\mid u(\rho(t))-u(s)-u^{\nabla}(t)(\rho(t)-s|\leq \varepsilon| \rho(t)-s \mid,
$$

for all $s \in U$.

We present here the necessary definitions of the theory of cones in Banach spaces and the Avery-Peterson fixed point theorem.

Definition 2.1. Let $E$ be a real Banach space over $R$. A nonempty convex closed set $P \subset E$ is said to be a cone provided that:

(1) $a u \in P$, for all $u \in P, a \geq 0$;

(2) $u,-u \in P$ implies $u=0$.

Definition 2.2. An operator is called completely continuous if it is continuous and maps bounded sets into pre-compact sets.

Definition 2.3. The map $\alpha$ is said to be a nonnegative continuous convex functional on a cone $P$ of a real Banach space $E$ provided that $\alpha: P \rightarrow[0,+\infty)$ is continuous and

$$
\alpha(t x+(1-t) y) \leq t \alpha(x)+(1-t) \alpha(y), \quad \text { for all } x, y \in P, \quad t \in[0,1] .
$$

Definition 2.4. The map $\beta$ is said to be a nonnegative continuous concave functional on a cone $P$ of a real Banach space $E$ provided that $\beta: P \rightarrow[0,+\infty)$ is continuous and

$$
\beta(t x+(1-t) y) \leq t \beta(x)+(1-t) \beta(y), \quad \text { for all } x, y \in P, \quad t \in[0,1] .
$$

Let $\gamma, \theta$ be nonnegative continuous convex functionals on $P, \alpha$ be a nonnegative continuous concave functional on $P$ and $\psi$ be a nonnegative continuous functional on $P$. Then for positive numbers $a, b, c$ and $d$, we define the following convex sets:

$$
\begin{aligned}
& P(\gamma, d)=\{x \in P \mid \gamma(x)<d\}, \\
& P(\gamma, \alpha, b, d)=\{x \in P \mid b \leq \alpha(x), \gamma(x) \leq d\}, \\
& P(\gamma, \theta, \alpha, b, c, d)=\{x \in P \mid b \leq \alpha(x), \theta(x) \leq c, \gamma(x) \leq d\},
\end{aligned}
$$

and a closed set

$$
R(\gamma, \psi, a, d)=\{x \in P \mid a \leq \psi(x), \gamma(x) \leq d\} .
$$

Lemma 2.1. [16] Let $P$ be a cone in Banach space E. Let $\gamma, \theta$ be nonnegative continuous convex functionals on $P, \alpha$ be a nonnegative continuous concave functional on $P$, and $\psi$ be a nonnegative continuous functional on $P$ satisfying 


$$
\psi(\lambda x) \leq \lambda \psi(x), \quad \text { for } 0 \leq \lambda \leq 1,
$$

such that for some positive numbers $l$ and $\mathrm{d}$,

$$
\alpha(x) \leq \psi(x), \quad\|x\| \leq l \gamma(x)
$$

for all $x \in \overline{P(\gamma, d)}$. Suppose $T: \overline{P(\gamma, d)} \rightarrow \overline{P(\gamma, d)}$ is completely continuous and there exist positive numbers $a, b, c$ with $a<b$ such that

(S1) $\{x \in P(\gamma, \theta, \alpha, b, c, d) \mid \alpha(x)>b\} \neq \varnothing$ and $\alpha(T x)>b$ for $x \in P(\gamma, \theta, \alpha, b, c, d)$;

$\left(S_{2}\right) \alpha(T x)>b$ for $x \in P(\gamma, \alpha, b, d)$ with $\theta(T x)>c$;

$\left(S_{3}\right) 0 \notin R(\gamma, \psi, a, d)$ and $\psi(T x)<a$ for $x \in R(\gamma, \psi, a, d)$ with $\psi(x)=a$.

Then $T$ has at least three fixed points $x_{1}, x_{2}, x_{3} \in \overline{P(\gamma, d)}$ such that:

$$
\begin{aligned}
\gamma\left(x_{i}\right) & \leq d, i=1,2,3 ; \\
b<\alpha\left(x_{1}\right) ; a & <\psi\left(x_{2}\right), \alpha\left(x_{2}\right)<b ; \\
\psi\left(x_{3}\right) & <a .
\end{aligned}
$$

\section{Positive solutions for problem $(1.5,1.6)$}

Lemma 3.1. [13] Suppose that condition $\left(H_{1}\right)$ holds, then the boundary value problem

$$
\begin{aligned}
& \left(\phi\left(u^{\Delta}\right)\right)^{\nabla}+h(t)=0, \quad t \in(0, T) \\
& u(0)=\sum_{i=1}^{m-2} \alpha_{i} u\left(\xi_{i}\right), \phi\left(u^{\Delta}(T)\right)=\sum_{i=1}^{m-2} \beta_{i} \phi\left(u^{\Delta}\left(\xi_{i}\right)\right),
\end{aligned}
$$

has the unique solution

$$
u(t)=\int_{0}^{t} \phi^{-1}\left(\int_{0}^{T} h(\tau) \nabla \tau-A\right) \Delta s+B
$$

where

$$
A=-\frac{\sum_{i=1}^{m-2} \beta_{i} \int_{\xi_{i}}^{T} h(\tau) \nabla \tau}{1-\sum_{i=1}^{m-2} \beta_{i}}, B=\frac{\sum_{i=1}^{m-2} \alpha_{i} \int_{0}^{\xi_{i}} \phi^{-1}\left(\int_{s}^{T} h(\tau) \nabla \tau-A\right) \Delta s}{1-\sum_{i=1}^{m-2} \alpha_{i}}
$$

Lemma 3.2. Suppose that condition $\left(H_{1}\right)$ holds, for $h \in C_{l d}[0, T]$ and $h(t) \geq 0$, the unique solution of problem $(3.1,3.2)$ satisfies

(1) $u(t) \geq 0, t \in[0, T]$.

(2) $\inf _{t \in[0, T]} u(t) \geq \delta \max _{t \in[0, T]}|u(t)|$, where

$$
\delta=\frac{\sum_{i=1}^{m-2} \alpha_{i} \xi_{i}}{\left(\left(1-\sum_{i=1}^{m-2} \alpha_{i}\right) T+\sum_{i=1}^{m-2} \alpha_{i} \xi_{i}\right)} .
$$

(3) $\max _{t \in[0, T]}|u(t)| \leq l \max _{t \in[0, T]_{T^{k}}}\left|u^{\Delta}(t)\right|$, where

$$
l=\frac{\left(\left(1-\sum_{i=1}^{m-2} \alpha_{i}\right) T+\sum_{i=1}^{m-2} \alpha_{i} \xi_{i}\right)}{\left(1-\sum_{i=1}^{m-2} \alpha_{i}\right)} .
$$


Proof. Parts (1) and (2) were established in [13]. We give the proof of (3). It is easy to check that

$$
\max _{t \in[0, T]}|u(t)|=u(T), \min _{t \in[0, T]}|u(t)|=u(0) .
$$

For the concavity of $u$ and the boundary condition, we get

$$
\left(1-\sum_{i=1}^{m-2} \alpha_{i}\right) u(0) \leq \sum_{i=1}^{m-2} \alpha_{i} \max \left|u^{\Delta}(t)\right| .
$$

This together with conclusion (2) ensures that conclusion (3) is satisfied.

Let $\mathrm{E}$ be the real Banach space $E=C^{\Delta}[0, \sigma(T)]$ to be the set of all $\Delta$-differential functions with continuous $\Delta$-derivative on $[0, \sigma(T)]$ with the norm

$$
\|u(t)\|_{1, T}=\max \left\{\|u\|_{0, T}\left\|u^{\Delta}\right\|_{0, T^{k}}\right\}
$$

where

$$
\begin{aligned}
\|u\|_{0, T} & =\sup \{|u(t)|: t \in[0, T]\} \\
\|u\|_{0, T^{k}} & =\sup \left\{\left|u^{\Delta}(t)\right|: t \in[0, T]_{T^{k}}\right\}, \quad u \in E .
\end{aligned}
$$

We define the cone $P \subset E$ by

$$
\begin{aligned}
& P=\left\{u \in E: u(t) \geq 0, u(0)=\sum_{i=1}^{m-2} \alpha_{i} u\left(\xi_{i}\right), \phi\left(u^{\Delta}(T)\right)=\sum_{i=1}^{m-2} \beta_{i} \phi\left(u^{\Delta}\left(\xi_{i}\right)\right),\right. \\
& u \text { is concave and increasing on }[0, T]\} \subset E \text {. }
\end{aligned}
$$

Let the nonnegative continuous concave functional $\alpha$, the nonnegative continuous convex functionals $\gamma, \theta$ and the nonnegative continuous functional $\psi$ be defined on the cone $P$ by

$$
\gamma(u)=\max _{t \in[0, T]_{T^{k}}}\left|u^{\Delta}(t)\right|, \theta(u)=\psi(u)=\max _{t \in[0, T]}|u(t)|, \alpha(u)=\min _{t \in[0, T]}|u(t)| .
$$

By Lemmas 3.3 and 3.4, the functionals defined above satisfy

$$
\delta \theta(x) \leq \alpha(x) \leq \theta(x)=\psi(x),\|x\|_{1, T} \leq l \gamma(x) .
$$

Therefore condition (2.2) of Lemma 2.1 is satisfied.

Define an operator $F: P \rightarrow E$ by

$$
\begin{aligned}
F u(t) & =\int_{0}^{t} \phi^{-1}\left(\int_{s}^{T} f\left(\tau, u(\tau), u^{\Delta}(\tau)\right) \nabla \tau+\frac{\sum_{i=1}^{m-2} \beta_{i} \int_{\xi_{i}}^{T} f\left(\tau, u(\tau), u^{\Delta}(\tau)\right) \nabla \tau}{1-\sum_{i=1}^{m-2} \beta_{i}}\right) \Delta s \\
& +\frac{1}{1-\sum_{i=1}^{m-2} \alpha_{i}}\left(\sum_{i=1}^{m-2} \alpha_{i} \int_{0}^{\xi_{i}} \phi^{-1}\left(\int_{s}^{T} f\left(\tau, u(\tau), u^{\Delta}(\tau)\right) \nabla \tau+\frac{\sum_{i=1}^{m-2} \beta_{i} \int_{\xi_{i}}^{T} f\left(\tau, u(\tau), u^{\Delta}(\tau)\right) \nabla \tau}{1-\sum_{i=1}^{m-2} \beta_{i}}\right) \Delta s\right)
\end{aligned}
$$

To present our main results, we assume there exist constants $0<a, b, c, d$ with $a<b$ $<d$ such that

$$
\left(A_{1}\right) f(t, u, v) \leq \frac{\phi(d)\left(1-\sum_{i=1}^{m-2} \beta_{i}\right)}{T-\sum_{i=1}^{m-2} \beta_{i} \xi_{i}},(t, u, v) \in[0,1]_{T} \times[0, l d] \times[-d, d]
$$


$\left(A_{2}\right) f(t, u, v)>\frac{\phi\left(b\left(1-\sum_{i=1}^{m-2} \alpha_{i}\right)\right)\left(1-\sum_{i=1}^{m-2} \beta_{i}\right)}{\phi\left(\sum_{i=1}^{m-2} \alpha_{i} \xi_{i} \phi^{-1}\left(\beta_{i}\left(T-\xi_{i}\right)\right)\right)},(t, u, v) \in[0,1]_{T} \times[b, b / \delta] \times[-d, d] ;$

$\left(A_{3}\right) f(t, u, v)<\frac{\phi(a)\left(1-\sum_{i=1}^{m-2} \beta_{i}\right)}{\left(T-\sum_{i=1}^{m-2} \beta_{i} \xi_{i}\right) \phi\left(T+\frac{\sum_{i=1}^{m-2} \alpha_{i} \xi_{i}}{1-\sum_{i=1}^{m-2} \alpha_{i}}\right)},(t, u, v) \in[0,1]_{T} \times[0, a] \times[-d, d]$.

Theorem 3.1. Under the assumptions $\left(A_{1}\right)-\left(A_{3}\right)$, the boundary value problem (1.5)-

(1.6) has at least three positive solutions $u_{1}, u_{2}, u_{3}$ satisfying

$$
\begin{gathered}
\max _{t \in[0,1]_{T}}\left|u_{i}^{\Delta}(t)\right| \leq d, \quad i=1,2,3 ; \\
b<\min _{t \in[0,1]_{T}}\left|u_{1}(t)\right| ; a<\max _{t \in[0,1]_{T}}\left|u_{2}(t)\right|, \min _{t \in[0,1]_{T}}\left|u_{2}(t)\right|<b ; \\
\max _{t \in[0,1]_{T}}\left|u_{3}(t)\right| \leq a .
\end{gathered}
$$

\section{Proof.}

It is easy to check that problem (1.5), (1.6) has a solution $u(t)$ if and only if $u$ is a fixed

point of operator $F$.

If $u \in \overline{P(\gamma, d)}$, then $\gamma(u)=\max _{t \in[0,1]_{T^{k}}}\left|u^{\Delta}(t)\right| \leq d$. Thus

$$
f\left(t, u(t), u^{\Delta}(t)\right) \leq \frac{\phi(d)\left(1-\sum_{i=1}^{m-2} \beta_{i}\right)}{T-\sum_{i=1}^{m-2} \beta_{i} \xi_{i}} .
$$

Then,

$$
\begin{aligned}
\gamma(T u) & =\phi^{-1}\left(\int_{s}^{T} f\left(t, u(t), u^{\Delta}(t)\right) \nabla t+\frac{\sum_{i=1}^{m-2} \beta_{i} \int_{\xi_{i}}^{T} f\left(t, u(t), u^{\Delta}(t)\right) \nabla \tau}{1-\sum_{i=1}^{m-2} \beta_{i}}\right) \\
& =\phi^{-1}\left(\frac{1}{1-\sum_{i=1}^{m-2} \beta_{i}}\right) \phi^{-1}\left(\int_{0}^{T} f\left(t, u(t), u^{\Delta}(t)\right) \nabla t-\sum_{i=1}^{m-2} \beta_{i} \int_{0}^{\xi_{i}} f\left(t, u(t), u^{\Delta}(t)\right) \nabla t\right) \\
& \leq d .
\end{aligned}
$$

Hence $F: \overline{P(\gamma, d)} \rightarrow \overline{P(\gamma, d)}$.

To check condition $\left(S_{1}\right)$ of Lemma 2.1, we choose $u(t) \equiv \frac{b}{\delta}=c$. It's easy to see $u(t)=\frac{b}{\delta} \in P(\gamma, \theta, \alpha, b, c, d)$ and $\alpha\left(\frac{b}{\delta}\right)>b$. So $\{u \in P(\gamma, \theta, \alpha, b, c, d \mid \alpha(x)>b)\} \neq \emptyset$.

If $u \in P(\gamma, \theta, \alpha, b, c, d)$, we have $b \leq u(t) \leq \frac{b}{\delta},\left|u^{\Delta}(t)\right| \leq d$. From assumption $\left(A_{2}\right)$, we have

$$
f\left(t, u(t), u^{\Delta}(t)\right) \geq \frac{\phi\left(b\left(1-\sum_{i=1}^{m-2} \alpha_{i}\right)\right)\left(1-\sum_{i=1}^{m-2} \beta_{i}\right)}{\phi\left(\sum_{i=1}^{m-2} \alpha_{i} \xi_{i} \phi^{-1}\left(\beta_{i}\left(T-\xi_{i}\right)\right)\right)} .
$$


Thus,

$$
\begin{aligned}
\alpha(F u) & =\frac{1}{1-\sum_{i=1}^{m-2} \alpha_{i}}\left(\sum_{i=1}^{m-2} \alpha_{i} \int_{0}^{\xi_{i}} \phi^{-1}\left(\int_{s}^{T} f\left(\tau, u(\tau), u^{\Delta}(\tau)\right) \nabla \tau+\frac{\sum_{i=1}^{m-2} \beta_{i} \int_{\xi_{i}}^{T} f\left(\tau, u(\tau), u^{\Delta}(\tau)\right) \nabla \tau}{1-\sum_{i=1}^{m-2} \beta_{i}}\right) \Delta s\right) \\
& \geq \frac{1}{1-\sum_{i=1}^{m-2} \alpha_{i}}\left(\sum_{i=1}^{m-2} \alpha_{i} \int_{0}^{\xi_{i}} \phi^{-1}\left(\frac{\sum_{i=1}^{m-2} \int_{\xi_{i}}^{T} f\left(\tau, u(\tau), u^{\Delta}(\tau)\right)}{1-\sum_{i=1}^{m-2} \beta_{i}}\right) \Delta s\right)>b,
\end{aligned}
$$

so $\alpha(F u)>b, \forall u \in P(\gamma, \theta, \alpha, b, b / \delta, d)$.

Second, with (4.1), we have $\alpha(F u) \geq \delta \theta(F u)>\delta b / \delta=b$ for all $u \in P(\gamma, \alpha, b, d)$ with $\theta(F u)>b / \delta$. Thus, condition $\left(S_{2}\right)$ of Lemma 2.1 is satisfied.

Finally we show that $\left(S_{3}\right)$ also holds. Clearly, as $\psi(0)=0<a$, we see $0 \notin R(\gamma, \psi, a, d)$. Suppose that $x \in R(\gamma, \psi, a, d)$ with $\psi(u)=a$, then assumption $\left(A_{3}\right)$ holds. then

$$
\begin{aligned}
& \psi(F u)=F(u(T))=\int_{0}^{T} \phi^{-1}\left(\int_{0}^{T} f\left(\tau, u(\tau), u^{\Delta}(\tau)\right) \nabla \tau+\frac{\sum_{i=1}^{m-2} \beta_{i} \int_{\xi_{i}}^{T} f\left(\tau, u(\tau), u^{\Delta}(\tau)\right) \nabla \tau}{1-\sum_{i=1}^{m-2} \beta_{i}}\right) \Delta s \\
& +\frac{1}{1-\sum_{i=1}^{m-2} \alpha_{i}}\left(\sum_{i=1}^{m-2} \alpha_{i} \int_{0}^{\xi_{i}} \phi^{-1}\left(\int_{s}^{T} f\left(\tau, u(\tau), u^{\Delta}(\tau)\right) \nabla \tau+\frac{\sum_{i=1}^{m-2} \beta_{i} \int_{\xi_{i}}^{T} f\left(\tau, u(\tau), u^{\Delta}(\tau)\right) \nabla \tau}{1-\sum_{i=1}^{m-2} \beta_{i}}\right) \Delta s\right) \\
& <a .
\end{aligned}
$$

So we verify that condition $\left(S_{3}\right)$ of Lemma 2.1 is satisfied. Thus, an application of Lemma 2.1 implies that the boundary value problem (1.5)-(1.6) has at least three positive solutions $u_{1}, u_{2}, u_{3}$ satisfying (3.3).

\section{Positive solutions for problem $(1.5,1.7)$}

In this section, we present the existence of positive solutions for problem $(1.5,1.7)$.

Lemma 4.1. [15] Suppose that condition $\left(H_{1}\right)$ holds, then boundary value problem

$$
\begin{aligned}
& \left(\phi\left(u^{\Delta}\right)\right)^{\nabla}+h(t)=0, \quad t \in(0, T), \\
& \phi\left(u^{\Delta}(0)\right)=\sum_{i=1}^{m-2} \alpha_{i} \phi\left(u^{\Delta}\left(\xi_{i}\right)\right), u(T)=\sum_{i=1}^{m-2} \beta_{i} u\left(\xi_{i}\right),
\end{aligned}
$$

has the unique solution

$$
u(t)=\int_{t}^{T} \phi^{-1}\left(\int_{0}^{s} h(\tau) \nabla \tau-A_{1}\right) \Delta s+B_{1}
$$

where

$$
A_{1}=-\frac{\sum_{i=1}^{m-2} \alpha_{i} \int_{0}^{\xi_{i}} h(\tau) \nabla \tau}{1-\sum_{i=1}^{m-2} \alpha_{i}}, B_{1}=\frac{\sum_{i=1}^{m-2} \beta_{i} \int_{\xi_{i}}^{T} \phi^{-1}\left(\int_{0}^{s} h(\tau) \nabla \tau-A_{1}\right) \Delta s}{1-\sum_{i=1}^{m-2} \beta_{i}}
$$

Lemma 4.2. Suppose that condition $\left(H_{1}\right)$ holds, for $h \in C_{l d}[0, T]$ and $h(t) \geq 0$, the unique solution of problem $(4.1,4.2)$ satisfies 
(1) $u(t) \geq 0, t \in[0, T]$

(2) $\inf _{t \in[0, T]} u(t) \geq \delta_{1} \max _{t \in[0, T]}|u(t)|$, where $\delta_{1}=\left(\sum_{i=1}^{m-2} \beta_{i}\left(T-\xi_{i}\right)\right) / T$ is a constant.

(3) $\max _{t \in[0, T]}|u(t)| \leq \gamma \max _{t \in[0, T]_{T^{k}}}\left|u^{\Delta}(t)\right|$, where $\quad l_{1}=T /\left(1-\sum_{i=1}^{m-2} \beta_{i}\right)$. is $\quad$ a constant.

Proof. Parts (1) and (2) are established in [15]. It is easy to check that

$$
\max _{t \in[0, T]}|u(t)|=u(0), \min _{t \in[0, T]}|u(t)|=u(T) .
$$

For the concavity of $u$ and the boundary condition, we get

$$
\left(1-\sum_{i=1}^{m-2} \beta_{i}\right) u(T) \leq \sum_{i=1}^{m-2} \beta_{i}\left(T-\xi_{i}\right) \max \left|u^{\Delta}(t)\right| .
$$

This together with conclusion (2) ensures that conclusion (3) is satisfied. We define the cone $P_{1} \subset E$ by

$$
P_{1}=\left\{u \in E: u(t) \geq 0, \phi\left(u^{\Delta}(0)\right)=\sum_{i=1}^{m-2} \alpha_{i} \phi\left(u^{\Delta}\left(\xi_{i}\right)\right), u(T)=\sum_{i=1}^{m-2} \beta_{i} u\left(\xi_{i}\right),\right.
$$

$u$ is concave and decreasing on $[0, T]\} \subset E$.

Define an operator $G: P \rightarrow E$ by

$$
\begin{gathered}
G(u(t))=\int_{t}^{T} \phi^{-1}\left(\int_{0}^{s} f\left(\tau, u(\tau), u^{\Delta}(\tau)\right) \nabla \tau+\frac{\sum_{i=1}^{m-2} \alpha_{i} \int_{0}^{\xi_{i}} f\left(\tau, u(\tau), u^{\Delta}(\tau)\right) \nabla \tau}{1-\sum_{i=1}^{m-2} \alpha_{i}}\right) \Delta s \\
+\frac{1}{1-\sum_{i=1}^{m-2} \beta_{i}}\left(\sum_{i=1}^{m-2} \beta_{i} \int_{\xi_{i}}^{T} \phi^{-1}\left(\int_{0}^{s} f\left(\tau, u(\tau), u^{\Delta}(\tau)\right) \nabla \tau+\frac{\sum_{i=1}^{m-2} \alpha_{i} \int_{0}^{\xi_{i}} f\left(\tau, u(\tau), u^{\Delta}(\tau)\right) \nabla \tau}{1-\sum_{i=1}^{m-2} \alpha_{i}}\right) \Delta s\right)
\end{gathered}
$$

To present our main results, we assume there exist constants $0<a_{1}, b_{1}, c_{1}, d$ with $a_{1}$ $<b_{1}<d_{1}$ such that

$\left.\mathrm{A}_{4}\right) f(t, u, v) \leq \frac{\phi\left(d_{1}\right)\left(1-\sum_{i=1}^{m-2} \alpha_{i}\right)}{T-\sum_{i=1}^{m-2} \alpha_{i}\left(T-\xi_{i}\right)},(t, u, v) \in[0,1]_{T} \times\left[0, l_{1} d_{1}\right] \times\left[-d_{1}, d_{1}\right]$

$\left.\mathrm{A}_{5}\right) f(t, u, v)>\frac{1}{\sum_{i=1}^{m-2} \alpha_{i} \xi_{i}} \phi\left(\frac{b_{1}\left(1-\sum_{i=1}^{m-2} \beta_{i}\right)}{\sum_{i=1}^{m-2} \beta_{i}\left(T-\xi_{i}\right)}\right),(t, u, v) \in[0,1]_{T} \times\left[b_{1}, b_{1} / \delta_{1}\right] \times\left[-d_{1}, d_{1}\right]$;

A $) f(t, u, v)<\phi\left(\frac{1-\sum_{i=1}^{m-2} \beta_{i}}{T-\sum_{i=1}^{m-2} \beta_{i} \xi_{i}}\right) \frac{1-\sum_{i=1}^{m-2} \alpha_{i}}{T+\sum_{i=1}^{m-2} \alpha_{i}\left(T-\xi_{i}\right)} \phi\left(a_{1}\right),(t, u, v) \in[0,1]_{T} \times\left[0, a_{1}\right] \times\left[-d_{1}, d_{1}\right]$.

Theorem 4.1. Under the assumptions $\left(A_{4}\right)-\left(A_{6}\right)$, the boundary value problem (1.5),

(1.7) has at least three positive solutions $u_{1}, u_{2}, u_{3}$ satisfying

$$
\begin{gathered}
\max _{t \in[0,1]_{T}}\left|u_{i}^{\Delta}(t)\right| \leq d_{1}, \quad i=1,2,3 ; \\
b<\min _{t \in[0,1]_{T}}\left|u_{1}(t)\right| ; a_{1}<\max _{t \in[0,1]_{T}}\left|u_{2}(t)\right|, \min _{t \in[0,1]_{T}}\left|u_{2}(t)\right|<b_{1} ; \\
\max _{t \in[0,1]_{T}}\left|u_{3}(t)\right| \leq a_{1} .
\end{gathered}
$$

The proof of Theorem 4.1 is similar with the Theorem 3.1 and is omitted here. 


\section{Acknowledgements}

This study was supported by the Anhui Provincial Natural Science Foundation (10040606Q50), National Natural Science Foundation of China (No. 11071164), Shanghai Natural Science Foundation (No. 10ZR1420800) and Shangha Leading Academic Discipline Project (No. S30501).

\section{Author details}

${ }^{1}$ College of Science, University of Shanghai for Science and Technology, Shanghai, 200093, P.R. China ${ }^{2}$ Department of Mathematics, Hefei Normal University, Hefei 230601, P.R. China

\section{Authors' contributions}

The authors declare that the work was realized in collaboration with the same responsibility. All authors read and approved the final manuscript.

\section{Competing interests}

The authors declare that they have no competing interests.

Received: 31 August 2011 Accepted: 15 February 2012 Published: 15 February 2012

\section{References}

1. Ma, R: Positive solutions for a nonlinear three-point boundary value problem. Electron J Diff Equ. 34, 1-8 (1999)

2. Ma, R, Cataneda, N: Existence of solution for nonlinear m-point boundary value problem. J Math Anal Appl. 256, 556-567 (2001). doi:10.1006/jmaa.2000.7320

3. Bai, Z, Ge, W, Wang, Y: Multiplicity results for some second-order four-point boundary-value problems. Nonlinear Anal. 60, 491-500 (2004)

4. Yang, L, Liu, X, Jia, M: Multiplicity results for second-order m-point boundary value problem. J Math Anal Appl. 324, 532-542 (2006). doi:10.1016/j.jmaa.2005.07.084

5. Chen, $\mathrm{S}, \mathrm{Hu}, \mathrm{J}$, Chen, $\mathrm{L}$, Wang, C: Existence for $n$-point boundary value problem of second order ordinary differential equations. J Comput Appl Math. 180, 425-432 (2005). doi:10.1016/..cam.2004.11.010

6. Atici, F, Cabada, A: Existence and uniqueness results for discrete second-order periodic boundary value problem. Comput Math Appl. 45, 1417-142 (2003). doi:10.1016/S0898-1221(03)00097-X

7. Cabada, A, Otero-Espinar, V: Optimal existence results for $n$-order periodic boundary value difference problems. J Math Anal Appl. 247, 67-86 (2000). doi:10.1006/jmaa.2000.6824

8. Atici, F, Guseinov, G: On Green's functions and positive solutions for boundary value problems on time scales. J Comput Appl Math. 141, 75-99 (2002). doi:10.1016/S0377-0427(01)00437-X

9. Agarwal, R, Otero-Espinar, V, Perera, K: Multiple positive solutions of singular dirichlet problems on time scales via variational methods. Nonlinear Anal 67, 368-381 (2007). etc. doi:10.1016/j.na.2006.05.014

10. Chang, $Y, L i, W$ : Existence results for second-order dynamic inclusion with $m$-point boundary value conditions on time scales. Appl Math Lett. 20, 885-891 (2007). doi:10.1016/j.aml.2006.09.004

11. Wang, $\mathrm{PG}, \mathrm{Wu}, \mathrm{HX}, \mathrm{Wu}, \mathrm{Y}$ : Higher even-order convergence and coupled solutions for second-order BVPs on time scales. Comput Math Appl. 55, 1693-1705 (2008). doi:10.1016/j.camwa.2007.06.026

12. Wang, $P G, W u, M$ : Practical $\bigotimes_{0}$-stability of impulsive dynamic system on time scales. Appl Math Lett. 20(6):651-658 (2007). doi:10.1016/j.aml.2006.06.016

13. Han, W, Jin, Z, Kang, S: Existence of positive solutions of nonlinear m-point BVP for an increasing homeomorphism and positive homomorphism on time scales. J Comput Appl Math. 233, 188-196 (2009). doi:10.1016/j.cam.2009.07.009

14. Han, W, Jin, Z: Positive solutions of nonlinear $m$-point BVP for an increasing homeomorphism and positive homomorphism with sign changing nonlinearity on time scales. Commun Nonlinear Sci Numer Simul. 15, 690-699 (2010). doi:10.1016/j.cnsns.2009.04.012

15. Sang, $Y, S u, H, X u$, F: Positive solutions of nonlinear $m$-point BVP for an increasing homeomorphism and homomorphism with sign changing nonlinearity on time scales. Comput Math Appl. 58, 216-226 (2009). doi:10.1016/j. camwa.2009.03.106

16. Avery, R, Peterson, A: Three positive fixed points of nonlinear operators on an ordered Banach space. Comput Math Appl. 208, 313-322 (2001)

doi:10.1186/1687-1847-2012-13

Cite this article as: Yang and Zhang: Multi-point boundary value problems for an increasing homeomorphism and positive homomorphism on time scales. Advances in Difference Equations 2012 2012:13. 